\title{
WIDEBAND BANDPASS FILTER DESIGN BASED ON RF-MEMS TECHNOLOGY
}

\author{
Syed M. Sifat ${ }^{1}$, Raj Savaj ${ }^{2}$, Ion Stiharu ${ }^{2}$, Ahmed Kishk ${ }^{1}$ \\ ${ }^{1}$ Concordia University, ECE Department, Montreal, QC, Canada \\ ${ }^{2}$ Concordia University, MIAE Department, Montreal, QC, Canada \\ E-mails: s_sifat@encs.concordia.ca, istih@encs.concordia.ca
}

\begin{abstract}
In this paper, we shall present the design steps and analyze the performance of a wideband band pass filter based on RF MEMS technology. MEMS technology enables very accurate features, which enables the repeatability of the filter. The filter is configured as a parallel ledge coupled five-pole micro strip wideband band pass filter. The concept consists of using precisely sized cantilever beams to excite the filters, which that will act as a switch to feed the filter. The bimetallic switch uses a cantilever beam to perform the deflection based on temperature rise. The filter part is designed using CST Microwave Studio (Frequency Domain Solver), and the cantilever is amiss designee dosing AUTOCAD.
\end{abstract}

Keywords: RF-MEMS, Cantilever Beam, Band Pass Filter, Bimetallic Switch.

\section{Introduction}

Micro electromechanical systems (MEMS) have gained its prominence and becoming very attractive in displaying superior performance in building compact devices and components. Micro electromechanical systems (MEMS) are miniature devices combining electrical and mechanical components and fabricated using integrated circuits (IC) batch-processing techniques $[1,2]$. In terms of fabrication, the surface micromachining and bulk micromachining are the most commonly used techniques. Surface micromachining consists of the deposition and lithographic patterning of various thin films, usually on silicon substrates as illustrated in Fig. 1. The deposition can be performed by adding a "sacrificial film" underneath the one which needs to be released and removed in the last steps of the process by selective etching techniques. The variety of structural materials is enormous, including many metals ( $\mathrm{Au}, \mathrm{Al}$, etc.), ceramics ( $\mathrm{SiO} 2$ and $\mathrm{Si} 3 \mathrm{~N} 4)$, and plastics such as photo resist, polymethyl methacrylate (PMMA), and others [3].

On the other hand, for bulk micromachining, a silicon substrate wafer is selectively etched to create the structures. The process includes the steps of wet chemical etching, reactive-ion etching (RIE), or both to form the released or fixed microstructures. With wet etching, the resulting structures depend on the directionality of the etching, which is a function of the crystalline of the substrate and the etching chemistry [4]. Fig. 2 illustrates the bulk micromachining technique with all the required fabrication steps.

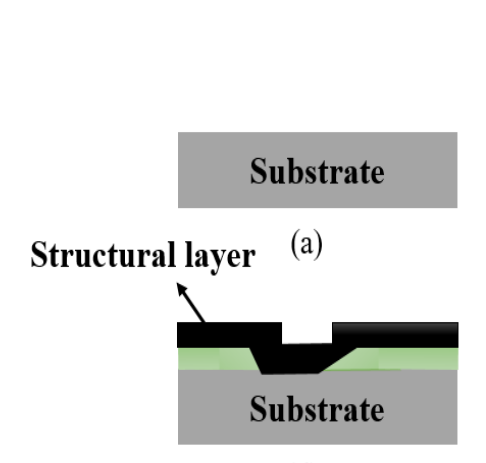

(d)

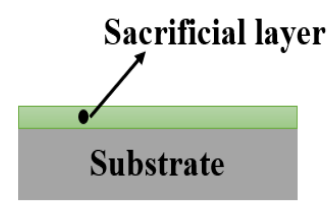

(b)

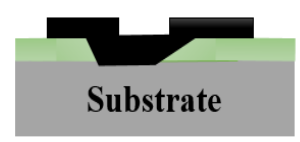

(e)

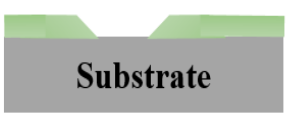

(c)

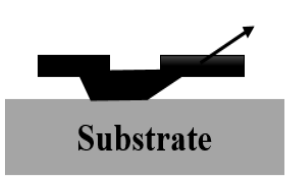

(f)

Figure 1: Typical Steps in a surface micromachining process, (a) Silicon substrate, (b) Deposition of sacrificial layer, (c) Etching sacrificial layer, (d) Deposition of the structural layer, (e) Shape definition by patterning and etching, (f) Release of suspended structure. 


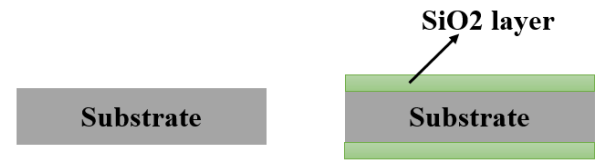

(b)

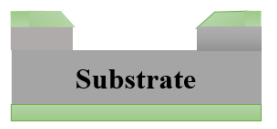

(d)

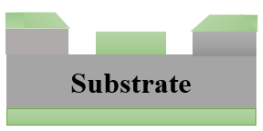

(e)

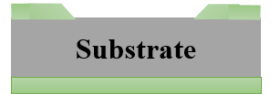

(c)

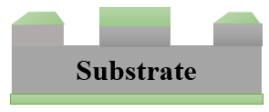

(f)

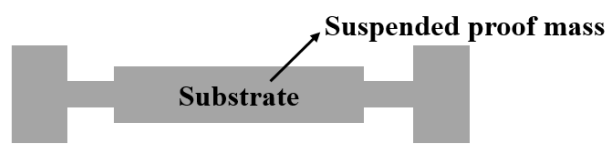

(g)

Figure 2: Typical steps in a bulk micromachining process (dry etching): (a) Silicon substrate preparation, (b) Deposition of $\mathrm{SiO}_{2}$ layer, (c) Etching of $\mathrm{SiO}_{2}$ layer (hard mask, when necessary), (d) Substrate etching, (e) Deposition of $\mathrm{SiO}_{2}$ layer for a selective area, (f) Substrate etching, ( $g$ ) Creation of suspended structure. The figure shows from a) to f) only one side patterning. The bottom could be processed simultaneous with the top or one side at the time to achieve the geometry as in (g).

Micro electromechanical Systems (MEMS) have been developed and improved since the early 1970's for various application purposes such as pressure and temperature measurement, as accelerometers, gas sensors, radiation sensors and others. MEMS switches for low-frequency applications have been deployed in the early 1980's.

Later, in the beginnings of 1990's, MEMS switches were developed for microwave applications [5]. MEMS devices are now used in large fields of applications including automotive, biomedical, consumer, aerospace and military applications. In wireless communication devices, RF MEMS devices such as phase shifters, filters, on-chip antennas, and a tenable matching network for wideband radios are generally used [6].

The RF MEMS switches are ideal for low power reconfigurable networks and subsystems, given the low insertion loss, and high Q-factor. RF switches are easily integrated with antennas, filters, and low-loss matching networks [7-11]. The current CMOS substrate technologies are not suited for integrating antennas; the silicon substrate has a low resistivity in the range of $10 \Omega-\mathrm{cm}$ - which is beneficial for the IC design, while it prevents latch-up currents. However, once the antenna radiates, most of the energy will pass by the low resistivity substrate, and will be absorbed/dissipated, rather than radiating to the free space [12].

In addition, a high amount of surface waves will travel in the substrate that might increase the losses.

Recent developments in micromachining techniques have resulted in novel high performance, low-loss filters for microwave and millimetre-wave applications [13-16]. There are different types of micro-machined filters. One particular example is based on the concept of suspending the micro strip or strip-line on thin dielectric membranes (typically $1.5 \mu \mathrm{m}$ thick) to eliminate dielectric loss and dispersion problems, resulting in a pure TEM
(Transverse Electro Magnetic) mode of propagation and conductor-loss limited performance [14]. In addition, a membrane fabrication and cavity formation, a three-layer structure of SiO2-Si3N4SiO2 is deposited on a high-resistivity silicon substrate using thermal oxidation and hightemperature chemical vapour deposition [15]. Park et al. [16] developed a tenable mm-wave band pass filter using cantilever-based $\mathrm{Au}$ varactors, which produces a tuning range of $0.8 \mathrm{GHz}(2.5 \%)$ at $32 \mathrm{GHz}$. The filter was built on a glass substrate, and it was based on a lumped element design.

In this work, we will present the design steps and perform the performance analysis of a parallel edgecoupled band pass filters in RF-MEMS technology, which can work for UWB (Ultra-Wide Band) applications. For the simulation of the band pass filter, we have used CST MICROWAVE STUDIO of Dassault Systems. The band pass filter can be excited with the help of a bimetallic switch that uses a micro-cantilever beam to perform the deflection based on the rise in temperature. The cantilever beam is fixed at one end and free at the other end.

The organization of the paper is as follows: Section II describes a detailed description of the proposed filter. This will follow by the cantilever beam design and analysis in Section III.

Finally, Section IV provides a summary of the contributions of the paper.

\section{Parallel Edge-Coupled Five-pole Band pass Filter}

Figure 3 illustrates the schematic of the proposed parallel end-coupled band pass filter.

The band pass filter is designed using CST Microwave Studios, which uses a Finite Element Method (FEM) solver. 
The filter is printed on a PCB material having a dielectric constant, Eroof 10.2 , with a thickness of $\mathrm{h}=$ $0.15 \mathrm{~mm}$. In general, the parallel-coupled micro strip band pass filters use half-wavelength line resonators. All the elements are positioned so that adjacent resonators are parallel to each other along half of their length. This parallel arrangement gives relatively large coupling for a given spacing between resonators. Thus, filter structure is particularly convenient for wide bandwidth as compared to the structure with end-coupled micro strip filters.

The proposed band pass filter was designe dusing reference [17], pp.127-129. It is worth mentioning, and the sole reason behind choosing five elements is for obtaining a wide bandwidth.

Chebyshev transformer is one of the most convenient matching transformers, that provides an excellent matching bandwidth.

The parallel coupled band pass filter is planar, compact, easy to fabricate, and can be considered for the full-duplex communication in the wireless transmitter, and receivers end. It is also possible to integrate this kind of filter with some other existing guiding structure like Ridge gap waveguide technology where the propagation medium is air instead of a dielectric medium [18, 19].

Figure 4 illustrates the S-parameters of the proposed filter. The reflection coefficient S11 of the band pass filter is covering $15.7 \%$ impedance bandwidth from 34.2-40.1 GHz.

The insertion loss S21 is around $-0.6 \mathrm{~dB}$, which is considered very suitable in terms of transmitting power from one end to the other end.

At the frequency above and below the resonance the band pass filter would not allow any signal to pass, which is clearly visible from the S-parameter as above mentioned, is illustrated in Fig. 4.

It is important to point out that a waveguide port represents the ideal scenario for solving an electromagnetic problem; however, the accurate way to solve it is by using a co-axial connector.

Waveguide ports represent a special kind of boundary condition of the calculation domain, enabling the stimulation as well as the absorption of energy.

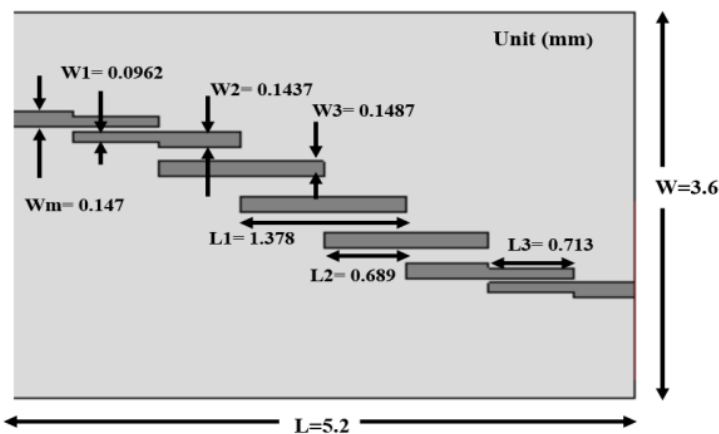

(a)

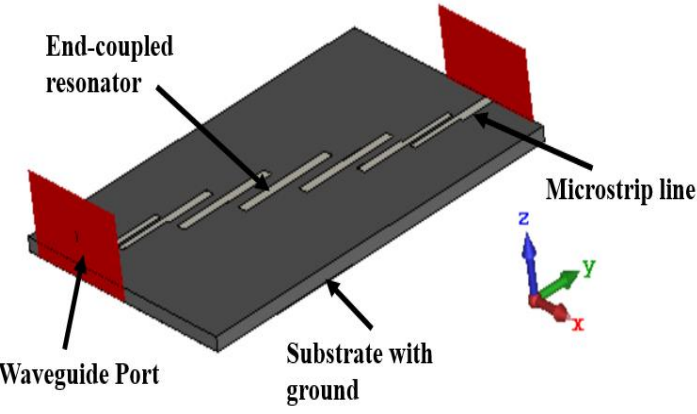

(b)

Figure 3: Five-element bandpass filter (a) schematic with required parameters, (b) 3D Geometry.

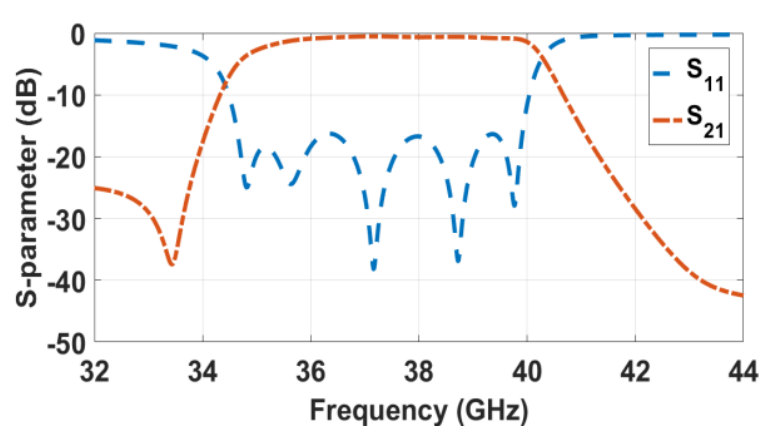

Figure 4: Scattering (S) parameters of the parallel end-coupled band pass filter with five elements.

Figure 5 illustrates the Electric field distribution of the band pass filter at four different frequencies. It is evident from the figure that at 34 and $42 \mathrm{GHz}$, which are on the top-bands, the filter is not allowing any signal to pass from Port 1 to Port 2 . Within the band pass frequency range, the coupling is strong between the input and the outputs through the parallel-coupled resonators.

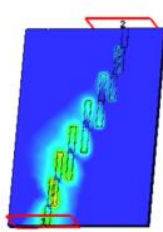

(a) $34 \mathrm{GHz}$

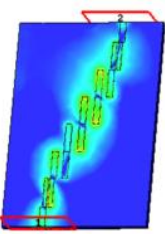

(b) $36 \mathrm{GHz}$

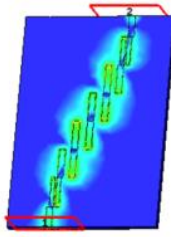

(c) $38 \mathrm{GHz}$

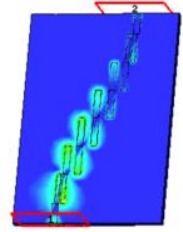

(d) $42 \mathrm{GHz}$
Figure 5: Electric (E) field distribution at various frequencies of the band pass filter.

\section{Cantilever Beam Design- Bimetallic Switch}

MEMS bimetallics witch isused given its ability to deflect when the temperature changes, which occurs without any mechanical force. The function of this switch is to control the openness and closeness of the electrical circuit $[20,21]$. Fig. 6 shows a bimetallics witch model that is normally used in electrical circuits. MEMS bimetallic switch is one cantilever beam that is fixed at one end and free at the other end. 
MEMS bimetallic switch is made of two metals joined in a true metallurgical bond enabled by the physical chemical deposition of two dislike metals. When temperature changes, the beam which is the switch, bends due to the different thermal expansion coefficients of both materials.

The switch will bend towards the material having a lower thermal expansion coefficient. Here it is worth mentioning that the lower side (blue colour) material might be doped poly silicon while the upper side (green colour) might be any metallic material.

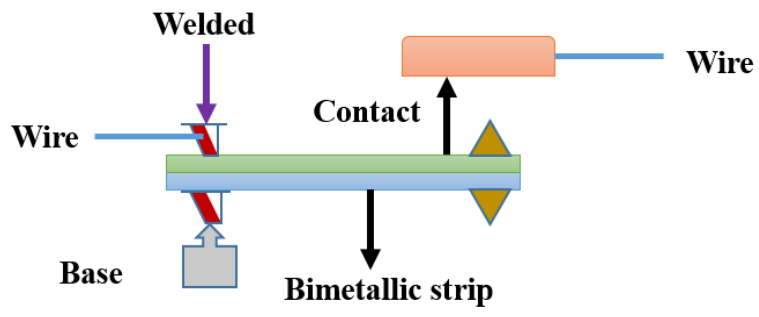

Figure 6: Bimetallic switch with cantilever beam in electrical circuit.

The width, length, and thickness, of the cantilever beam is $\mathrm{W}=0.147 \mathrm{~mm}, \mathrm{~L}=1.1 \mathrm{~mm}, \mathrm{~h}=0.01 \mathrm{~mm}$ respectively.

The poly silicon and the metallic layers are both kept at the thickness of the filter $\boldsymbol{h}_{S i}=h_{A I}=0.005$ $\mathrm{mm}$.

The selected sizes will enable a reliable operation of the system.

The design parameters of the designed bimetallic switch are illustrated in Table 1.
Table 1. Design parameters of the bimetallic switch

\begin{tabular}{|l|l|}
\hline Parameter & Value \\
\hline $\begin{array}{l}\text { Modulus of Elasticity of } \\
\text { Silicon, } E_{S i}\end{array}$ & $176 \mathrm{GPa}$ \\
\hline $\begin{array}{l}\text { Modulus of Elasticity of } \\
\text { Aluminum } E_{A l}\end{array}$ & $80 \mathrm{GPa}$ \\
\hline $\begin{array}{l}\text { Thermal expansion } \\
\text { coefficient of Silicon, } \alpha_{S i}\end{array}$ & $2.76 \times 10^{-6} k^{-1}$ \\
\hline $\begin{array}{l}\text { Thermal expansion } \\
\text { coefficient of aluminum, } \alpha_{A l}\end{array}$ & $23.1 \times 10^{-6} k^{-1}$ \\
\hline $\begin{array}{l}\text { Radius of beam after } \\
\text { deflection, } \mathrm{r}\end{array}$ & $6.8 \mathrm{~mm}$ \\
\hline $\begin{array}{l}\text { Curvature of beam after } \\
\text { deflection, } \mathrm{k}=1 / \mathrm{r}\end{array}$ & $0.1466 \mathrm{~mm}^{-1}$ \\
\hline Deflection of beam, y & $0.09 \mathrm{~mm}$ \\
\hline
\end{tabular}

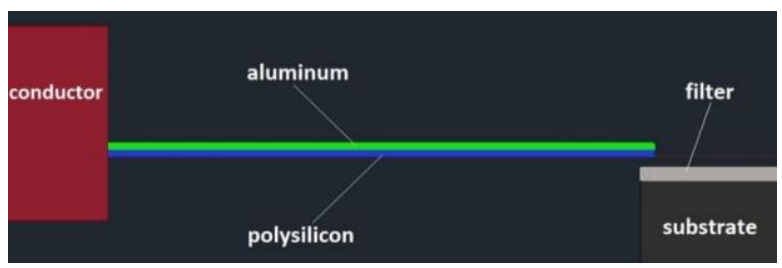

Figure 7: AutoCAD 2D geometry of bimetallic switch with filter

The AutoCAD model of the bimetallic switch model is illustrated in Fig. 7 with the cantilever beam. Assuming $50^{\circ} \mathrm{C}$ temperature rise for calculation, $\Delta \mathrm{T}=50^{\circ} \mathrm{C}$, the theoretical curvature equation is calculated using [21],

$$
k=\frac{6 E_{S} E_{A l}\left(h_{S i}+h_{A l}\right) h_{S i} h_{A l} \varepsilon}{E_{S i}{ }^{2} h_{S i}{ }^{4}+4 E_{S i} E_{A l} h_{S i}{ }^{3} h_{A l}+6 E_{S i} E_{A l} h_{S i}{ }^{2} h_{A l}{ }^{2}+4 E_{S i} E_{A l} h_{A l}{ }^{3} h_{S i}+E_{A l}{ }^{2} h_{A l}{ }^{4}}
$$

Where, $\varepsilon=\left(\alpha_{A l}-\alpha_{S i}\right) \Delta \mathrm{T}$

Curvature, $\mathrm{k}$ is related to deflection, $\mathrm{y}$ by the following equation,

$$
k=\frac{2 \sin \left(\tan ^{-1} y / l\right)}{\sqrt{l^{2}+y^{2}}}
$$

Hence, calculations show that the free end of this bimetallic switch deflects by $0.09 \mathrm{~mm}$ for a $50{ }^{\circ} \mathrm{C}$ temperature rise. It should be mention that these values are calculated using MATLAB coding. As Si has a lower thermal expansion coefficient than aluminum, so the beam as scaledwill bend $0.09 \mathrm{~mm}$ downside. Therefore, the beam will be positioned to touch the filterthat makes the circuit close and current flows through the filter.

One end of the beam is joined with the conductor, as illustrated in Fig. 7. When voltage is supplied, it will come through this conductive material. Material with a high resistivity requires small amount of current, and enough amount of heat is produced due to high resistivity, which will increase temperature. The more the temperature rise, the more beam bends. The heat produced by the current flow is $\mathrm{q}=$ $I^{2}$ Rt (Joule's equation of electric heating), where $I=$
Current $(A), R=$ Resistance $(\Omega)$, and $t=$ Time $(S)$. Conductive heat transfer per unit length is $\mathrm{q}=\mathrm{kA} \Delta T$ (according to Fourier's law) where $\mathrm{K}=$ Overall conductive heat transfer coefficient $(\mathrm{W} / \mathrm{mK}), \mathrm{A}=$ Cross-sectional area perpendicular to the current flow $\left(\mathrm{m}^{2}\right), \Delta T=$ Change in temperature $(\mathrm{k})$.

The deflection of the cantilever beam is calculated using MATLAB coding which is highlighted in Fig. 8.

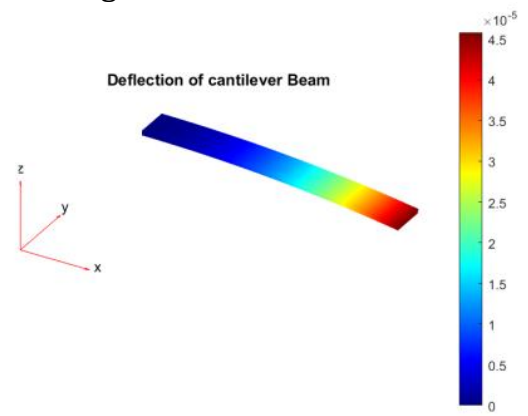

Figure 8: Deflection of the cantilever beam at $50^{\circ} \mathrm{C}$.

For $50^{\circ} \mathrm{C}$ increment in temparature the deflection is $0.09 \mathrm{~mm}$. The more temparature increases the more deflection will occur. For example, for $70^{\circ} \mathrm{C}$ 
temparature increament the deflection will be $0.12 \mathrm{~mm}$ which is more than the one encountered at $50^{\circ} \mathrm{C}$ as shown in Fig. 9.

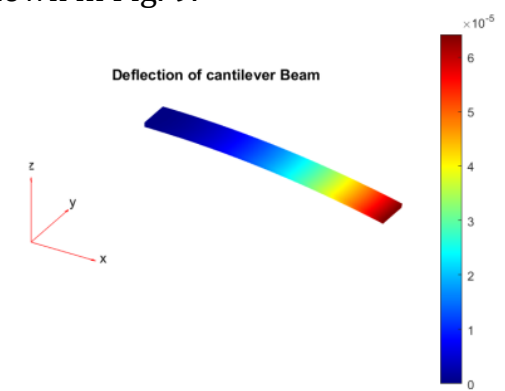

Figure 9: Deflection of the cantilever beam at $70^{\circ} \mathrm{C}$.

\section{Conclusion}

In this work, a wideband bandpass filter has been designed covering15.7\%impedancebandwidthfrom 34.2-40.1 GHz. The bandpass filter can be excited with the help of a bimetallic switch that uses a cantilever beam to perform the deflection based on the rise in temperature. This approach can be feasible for MEMS integrated circuit elements. It is possible to integrate cantilever beam switching with some other existing passive elements such as antennas, couplers, circulators, and power dividers from integrated circuits. Bandpass filters are widely used in wireless transmitter and receiver ends, and for ultrawideband full-duplex commutation.

\section{References}

[1] J. Bryzek, K. Peterson and W. McCulley, "Micro machines on the march," in IEEE Spectrum, vol. 31 , no. 5, pp. $20-31,(1994)$.

[2] E. R. Brown, "RF-MEMS switches for reconfigurable integrated circuits," IEEE Trans, MTT-46, pp. 1868-1880, (1998).

[3] K. E. Peterson, "Silicon as a mechanical material," Proc. IEEE, 70, pp. 420-457, (1982).

[4] M Gad-el-Hak, "Mems Introduction and Fundamentals,"2nd Edition, CRC Press, (2006).

[5] L. E. Larson, R. H. Hackett, M. A. Melendes and R. F. Lohr, "Micro machined microwave actuator (MIMAC) technology-a new tuning approach for microwave integrated circuits," IEEE Microwave and Millimeter-Wave Monolithic Circuits Symposium., Boston, MA, USA, pp. 27-30, (1991).

[6] Gabriel M. Rebeiz, "RF MEMS THEORY, DESIGN, AND TECHNOLOGY," Wiley, New Jersey, (2014).

[7] R. Brown and G. M. Rebeiz, "A high-performance integrated K-band diplexer," IEEE Trans. on Microwave Theory and Techniques, vol. 47, no. 8, pp. 1477-1481, (1999).

[8] R. C. Ruby et al., "High-Q FBAR filters in a waferlevel chipscale package," IEEE International Solid-State Circuits Conference, San Francisco, CA, USA, pp. 184-458, (2002).
[9] F. D. Bannon, J. R. Clark and C. T. Nguyen, "High-Q $\mathrm{HF}$ microelectromechanical filters" in IEEE Journal of Solid-State Circuits, vol. 35, no. 4, pp. 512-526, (2000).

[10] M. Kim, J. B. Hacker, R. E. Mihailovich and J. F. DeNatale, "A monolithic MEMS switched dualpath power amplifier," in IEEE Microwave and Wireless Components Letters, vol. 11, no. 7, pp. 285-286, (2001).

[11] E. Erdil, K. Topalli, M. Unlu, O. A. Civi and T. Akin, "Frequency Tunable Microstrip Patch Antenna Using RF MEMS Technology," IEEE Transactions on Antennas and Propagation, vol. 55, no. 4, pp. 1193-1196, (2007).

[12] Hammad M. Cheema and Atif Shamim, "The Last Barrier," IEEE Microwave Magazine, pp.79-91, (2013).

[13] G. M. Rebeiz, L. P. Katehi, T. M. Weller, C. Y. Chi, and S. V Robertson, "Micro machined Membrane filters for microwave and millimetre-wave applications," International Journal of Microwave Millimetre-Wave CAE, 7, pp. 149-166,(1997).

[14] T. M. Weller and L. P. Katehi, "Miniature stub and filter designs using the micro shield Transmission line," IEEE MTT-S, Digest, 675-678, (1995).

[15] P. Blondy, A. R. Brown, D. Cros, and G. M. Rebeiz, "Lowloss micro machined filters for Millimetrewave communication systems," in IEEE Trans., MTT-46, pp. 2283-2288, (1998).

[16] J-H Park, H-T Kim, Y. Kwon and Y.K- Kim "Tunable millimetre wave filters using coplanar waveguide an micro machined variable capacitors", Micro-engineering and Micromechanics, pp. 706-712, (2001).

[17] J.-S. Hong, M. J. Lancaster Micro strip Filters for RF/Microwave Applications, Hoboken, New Jersey: Wiley, (2001).

[18] M. Sharifi Sorkherizi and A. A. Kishk, "SelfPackaged, Low-Loss, Planar Band pass Filters for Millimetre-Wave Application Based on Printed Gap Waveguide Technology," in IEEE Transactions on Components, Packaging and Manufacturing Technology, vol. 7, no. 9, pp. 14191431, (2017).

[19] S. M. Sifat, M. M. M. Ali, S. I. Shams and A. Sebak, "High Gain Bow-Tie Slot Antenna Array Loaded With Grooves Based on Printed Ridge Gap Waveguide Technology," in IEEE Access, vol. 7, pp. 36177-36185, (2019).

[20] R. Al-Dahleh and R. R. Mansour, "A Novel Warped-Beam Design that Enhances RF Performance of Capacitive MEMS Switches,"IEEE/MTT-S International Microwave Symposium, Honolulu, HI, pp. 1813-1816, (2007).

[21] T. Clyne, "Residual stresses in surface coatings and their effects on interfacial debonding," Key Engineering Materials (Switzerland), vol. 116, (1996). 